\title{
Towards the Adoption of Cyber-Physical Systems of Systems Paradigm in Smart Manufacturing Environments
}

\author{
Borja Ramis Ferrer, Wael M. Mohammed, Jose L. \\ Martínez Lastra \\ FAST-Lab, Laboratory of Automation and Hydraulics \\ Tampere University of Technology \\ Tampere, Finland \\ \{borja.ramisferrer, wael.mohammed, jose.lastra\}@tut.fi
}

\author{
Alberto Villalonga ${ }^{1,2}$, Gerardo Beruvides ${ }^{1}$, Fernando \\ Castaño $^{1}$, Rodolfo E. Haber ${ }^{1}$ \\ ${ }^{1}$ Centre for Automation and Robotics (CAR-CSIC) \\ Madrid, Spain \\ \{gerardo.beruvides, fernando.castano, \\ rodolfo.haber\}@car.upm-csic.es \\ ${ }^{2}$ University of Matanzas (UM) \\ Matanzas, Cuba \\ alberto.villalonga@umcc.cu
}

\begin{abstract}
Cyber-physical Systems (CPS) in industrial manufacturing facilities demand a continuous interaction with different and a large amount of distributed and networked computing nodes, devices and human operators. These systems are critical to ensure the quality of production and the safety of persons working at the shop floor level. Furthermore, this situation is similar in other domains, such as logistics that, in turn, are connected and affect the overall production efficiency. In this context, this article presents some key steps for integrating three pillars of CPS (production line, logistics and facilities) into the current smart manufacturing environments in order to adopt an industrial Cyber-Physical Systems of Systems (CPSoS) paradigm. The approach is focused on the integration in several digital functionalities in a cloud-based platform to allow a real time multiple devices interaction, data analytics/sharing and machine learning-based global reconfiguration to increase the management and optimization capabilities for increasing the quality of facility services, safety and energy efficiency and industrial productivity. Conceptually, isolated systems may enhance their capabilities by accessing to information of other systems. The approach introduces particular vision, main components, potential and challenges of the envisioned CPSoS. In addition, the description of one scenario for realizing the CPSoS vision is presented. The results herein presented will pave the way for the adoption of CPSoS that can be used as a pilot for further research on this emerging topic.
\end{abstract}

Keywords- Industry 4.0; Cyber-Physical Systems; System of Systems; Cyber-Physical Systems of Systems; Smart Manufacturing.

\section{INTRODUCTION}

$\mathrm{T}$ he European manufacturing industry is looking for new methods that can empower the implementation and realization of digital transformation in the frame of Industry 4.0 paradigm [1, 2]. In the same direction, the European Commission is fostering the research of contemporary and novel technologies and methodologies for the interaction of systems [3, 4]. The deployment of validated solutions that enable not only the interaction of different-scale systems but also the integration of diverse domains is therefore mandatory.
From a high-level perspective, one target is the creation of an ecosystem formed by a System of Systems (SoS) [5, 6]. Conceptually, each system with their own capabilities feed other systems in order to create an integrated system, known as the SoS, with enhanced and even new functionalities [7-10].

In the following years, this should go beyond the interconnectivity and integration proposed e.g., by the Industry 4.0 [11-13] and smart cities [14]. This research work suggests the integration between more and yet fully-connected domain systems, such as logistics, building automation and production systems in order to increase the efficiency and productivity of industrial systems. Ultimately, this should create an interconnected ecosystem that permits the industry to predict and react to the high and dynamic market demands.

In this context, each individual system may be seen as an integration of Cyber and Physical Systems (CPS), as it can be seen in industrial and smart city systems $[15,16]$. Then, the created entity that integrates such isolated systems and enhance their functionalities will be a CPS of Systems (CPSoS). In other words, the CPSoS will interconnect the hardware, software and even people from unconnected systems. Then, the data generated by each individual component may impact on other sub-systems as it will contribute to the generation of the whole CPSoS knowledge information. However, the strategy to deal with complexity and heterogeneity of CPSoS is one of the main bottlenecks $[17,18]$.

The management, integration and computation of CPSoS data are some of the major challenges due to the heterogeneity of system resources which generates data [19]. This is currently solved through complex Information and Communication Technologies (ICT) based solutions in different domains, such as industrial automation and health care [20]. However, this research work describes other challenges that should be also considered before the CPSoS.

The main objective of this research work is to explore the ways for the effective adoption of CPSoS paradigms in smart 
manufacturing environments for enhancing the efficiency, data analytics, connectivity, multiple tasks execution, self-decision making and real time systems interaction. Then, the article presents the authors' vision and the main components that will be needed for the realization of CPSoS. Furthermore, potential and challenges are also addressed. In addition, the paper describes a real scenario of a CPSoS consists of 1) logistics, 2) building automation and 3 ) production systems.

The rest of the paper is structured as follows: Section II presents a theoretical background including the main areas in relation to the presented work. After that, Section III presents the essential steps toward the adoption of CPSoS by industry and the envisioned components, potential and challenges of the future implementation. Then, Section IV presents a conceptual scenario for proving the concept and the expected results. Finally, Section V concludes the work and describes the further work to be done.

\section{THEORETICAL BACKGROUND}

\section{A. Systems of systems for industrial applications}

As an emerging field, System of Systems (SoS) have no widely accepted definition. Nevertheless, one of the most completed definition was introduced by [21]: “... A system of systems is an integration of a finite number of constituent systems which are independent and operable, and which are networked together for a period of time to achieve a certain higher goal'.

SoS topologies can be classified into four main groups in function of the relationship among the constituents, the levels of responsibility and the authority level [22]: (1) virtual without central authority to manage activities; (2) collaborative, the constituent systems work together with different grades of voluntarily to fulfill agreed upon central purposes without engineering team to guide or manage SoS related activities; (3) directed, the constituent systems maintain their ability to operate independently, but evolution is predominantly controlled by SoS management organization and; (4) acknowledged, the objectives are organized, a manager is designated, and the resources are allocated at the SoS [23].

The broad development and integration of new technologies in modern industry has motivated a great interest in the design and implementation of SoS based on the potential offered in terms of production and services. This interest has also brought with the emergence number of challenges from the engineering point of view due to the high complexity, heterogeneity, organization and management's capability.

SoS have made possible to achieve great advances in new areas of research such as the smart grids, energy, mobility, industry and cities $[24,25]$. Technical and scientific literature shows several examples of development, coordination and integration framework such as: intelligent transportation operations in smart cities in the context of the Internet of Things [14]; the design of a proactive planning and optimizing methodology [26] and mechanism for choosing a coordinating strategy in smart grids [27]; and a disaster damages mitigation web-based user interface with support for GIS data representation [28].

In industrial environments, the SoS concept has been considered from the point of view of software design, management and exchange of information, as well as control techniques to optimize the production and management of physical processes [29, 30]. Many software-oriented methodologies have been developed such as: a multi-kind product life cycle software allows each system to possess operational and managerial independence proposed by [31] and the monitoring systems developed by [32] to support collecting, aggregating, analyzing and data representation in architecturally heterogeneous SoS implementations.

By the other hand, the demands of real-time, optimal and reliability solutions based on global information, knowledge and parametrization to execute reconfiguration and control actions introduce several challenges to the industrial SoS ecosystems [33]. This concept commonly introduces new elements to take into account in multi-agent interaction and dynamic control actions in order to make feasible selforganization and guarantee safety in scalability in smart scenarios, sharing information between a huge number of devices, sub-systems and ecosystems connected to each other $[34,35]$.

Finally, with the introduction of smart manufacturing and Industry 4.0 paradigm for digital transformation and the interconnectivity of multiple devices based on the Internet of Everything (ioE) and Internet of Things (IoT) concepts, as well as, the integration between cyber world (e.g., algorithms, software, apps, etc.) with the physical world (e.g., devices, machines, automobile, buildings, etc.) new synergies in the current industry has been established [36, 37]. In this context, the Cyber-Physical Systems of Systems (CPSoS) introduce a promise research and knowhow generation opportunities for the engineering community, government policy, society and industry of the future. The influence of the revolutionary advances on information technologies is significant in both areas, and thus it is not surprising that this kind of overlap exists [38].

\section{B. Industrial Cyber-physical systems}

The term Industry 4.0 stands for the fourth industrial revolution which is defined as a new level of digitalization, organization and control over the entire value chain of the products life cycle, traceability, machine-to-machine-to-human connectivity and individualized customer personalization [39]. Industry 4.0 found its bases in the new paradigms of information and communication technologies such as: Industrial Internet of things (IIoT), Industrial Cyber-Physical Systems (ICPS), Service-Oriented Architectures (SOA), cloud computing and big data implementations, as well as, the introduction of innovative advances in the cybersecurity, distribution and decentralization of the information and compute capabilities in new industrial connected ecosystems [40].

The combination of distributed computing systems in product and production system planning, engineering, with the utilization of data acquired during the manufacturing processes 
from sensors and actuators in order to perform condition based monitoring, control, knowledge generation, learning and reconfiguration actions are key functionalities of the current ICPS [41]. These characteristics are the basis to set the new generation of manufacturing standards known as smart manufacturing, extracting key information from the processes, products or services to ensure a global visualization, efficient management and knowledge representation [42]. This fusion between the cyber and physical world introduces new synergies to the current industrial ecosystems. Concepts such as: real-time condition monitoring, failure predictions, anomalies detection, diagnoses, cloud as a service and zero-defect productions are presented in Industry 4.0 strategies to increase the traceability, competiveness and personalization levels [43].

Furthermore, the introduction of cloud services, openmanufacturing platforms and crowdsourcing frameworks provides new insights (knowhow) to drive monitoring, execution, management and control in the industry of the future based on global information, self-learning, hyper-connectivity, and multiple devices real-time interaction, sharing information from one system to others in order to increase the connectivity, response capability and accessibility levels [44]. In these topics, an important contribution is introduced by the reference Architectural Model Industry 4.0 (RAMI 4.0), providing a global framework for the classification of technology in alignment with I4.0. The model combines three core dimensions of product development and production in a cuboidal space, covering from product to connection with global ecosystem and multiple layer integration from assessments to business unit, as well as, the interconnection of the shop floor devices with condition-based monitoring, Human-Machine Interfaces, open protocols and data analytics and management [45]. The dimension hierarchy levels are based on the layers defined in IEC 62264 and IEC 61512 [46].

Nevertheless, in the current manufacturing industry, the application of Manufacturing Execution Systems (MES) and Enterprise Resources Planning (ERP) are still very disseminated solutions to take into account visualization, planning and control tasks in several industrial scenarios [47, 48]. MES provides many functionalities to the current industry in relation to execute monitoring, resources allocation, tasks scheduling, data collection/acquisition, management, maintenance, tracking, performance analysis and control operations on the shop floor, with real-time access to key performance indices to facilitate the decision-making and reconfiguration actions [49]. By the other hand, ERP manages and track all the information and operational service in a company, covering functional areas such as: human resources, logistics, finance and production [50].

\section{CYBER-PHYSICAL SYSTEMS OF SYSTEMS FOR INDUSTRY: THE PROPOSAL}

\section{A. The vision}

The proposed Cyber-Physical Systems of Systems (CPSoS) is composed by three main systems which have direct impact on the quality and efficiency of industrial productivity. First, in the supply chain, last mile logistics is the least efficient stage and causes up to $28 \%$ of the total delivery cost [51]. On the other hand, the quality of facility services, as well as the commodity, safety and energy efficiency of facilities has a direct impact in factories. Thus, the second system to be integrated in the CPSoS is building automation systems. Finally, as the objective is the improvement of industrial productivity, the third type of system is the production system itself.

The high-level vision of the proposed CPSoS is shown in Figure 1. As a cyber and physical integration, the CPSoS is composed by different sub-systems and components from the two domains i.e., cyber and physical. These domains are connected by Internet of Everything (IoE) technologies.

First, the physical domain is composed by physical resources that are used at different systems for altering and/or sensing the environment. Examples of physical resources per CPSoS system are heaters for increasing the temperature of a building, robotic arms performing operations at factory shop floors and trucks transporting material from suppliers to manufacturers.

Secondly, the cyber domain is composed by a set of applications, including digital twins. Basically, the physical resources are represented by cyber models. These models facilitate the study of systems behavior in order to monitor their performance. In addition, this is relevant for the achievement of zero-defects manufacturing because makes possible to find anomalies on the basis of the virtual model and adjust the physical equipment [52].

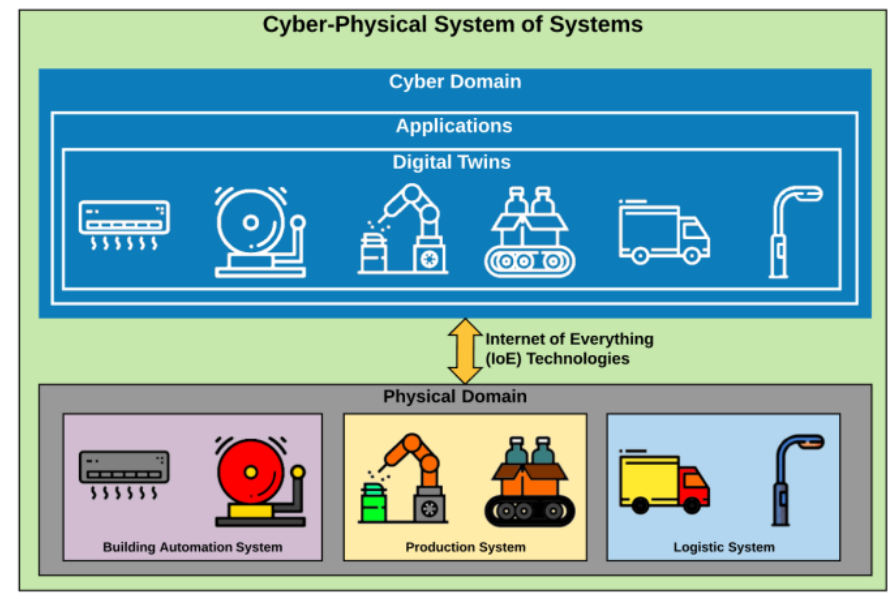

Figure 1. A high-level vision of the proposed CPSoS

\section{B. Main components of the approach}

Several components will be taken into account in the adoption of the proposed CPSoS scenario. The defined roadmap will address critical requests for the collection, integration, analysis and exchange of data and information, as well as the decision making in the entire ecosystem, becoming key components of the approach those that will permit the multiple systems interaction and share the data and information.

This research work proposes the collection of data within web-service enabled devices that are deployed in the different systems. On the other hand, such devices will be connected to both physical and cyber resources, such as sensors, actuators 
and data exchangers. In this context, human workers can also be considered as resources that will generate data within the CPSoS from the interaction with any sub-system, for example, sending a notification of the delivery of a package containing parts for production.

Furthermore, the interaction within CPSoS systems will need robust and flexible interfaces to ensure the management of heterogeneous data. As it will be further explained in the following subsection, the strategy to couple different data, information with different time scales is very challenging. One of the main components that will support the interaction within CPSoS systems will be the IoT devices [53]. These devices are currently deployed at factory shop floors in order to implement both horizontal and vertical integration of enterprise systems, following the SOA paradigm. Moreover, recent research reports [47] demonstrate the remote invocation of service operations for executing physical equipment actions. This may be extended to the collection of data from different data sources throughout different systems of the supply chain [43].

Finally, other important components of the CPSoS are the proprietary solutions used in different systems for different purposes, for instance, the application used by a client for ordering certain product or by a maintenance operator to report a malfunction at factory shop floor.

\section{Potential and challenges of the approach}

This research work identifies the potential of orchestrating isolated systems under a CPSoS. They can be presented as a set of qualitative attributes that will be critical for the CPSoS. The envisioned attributes are shown in Table I.

TABLE I. Potential of THE PROPOSED CPSOS

\begin{tabular}{|l|l|}
\hline $\begin{array}{l}\text { Qualitative } \\
\text { Attribute }\end{array}$ & Description \\
\hline Connectivity & $\begin{array}{l}\text { Current isolated systems that affect the productivity of } \\
\text { factories will be connected within IoT-based } \\
\text { implementations for collection and exchange of data. }\end{array}$ \\
\hline Digitalization & $\begin{array}{l}\text { Systems will be digitalized at Cyber world with digital } \\
\text { twins of resources in order to monitor and even control } \\
\text { their behavior }\end{array}$ \\
\hline Modelling & $\begin{array}{l}\text { Modelling techniques adopted by the CPSoS will help } \\
\text { to monitor systems behavior but also to suggest the } \\
\text { design of systems and process execution }\end{array}$ \\
\hline Flexible re- & $\begin{array}{l}\text { The analysis of huge amount of data produced by } \\
\text { physical resources and their digital models will permit } \\
\text { the the anticipation to required changes on runtime, } \\
\text { providing flexible re-configuration of systems }\end{array}$ \\
\hline $\begin{array}{l}\text { Versatility and } \\
\text { reusability }\end{array}$ & $\begin{array}{l}\text { The digital models will permit to analyze the } \\
\text { performance of systems in different situations, allowing } \\
\text { the assignment of new tasks not considered in design } \\
\text { phase. This will increment the reusability of systems' } \\
\text { components }\end{array}$ \\
\hline
\end{tabular}

Above potential attributes of the CPSoS leads to certain technological challenges that will be faced at development and deployment phases. Among them, the implementation of the proposes CPSoS will be challenging from the perspectives included in Table II.
Table I indicates that, mainly, the implementation of CPSoS will have problems related with the integration of the isolated systems and security. The employment of standard formats, as well as risk and threat modelling [54] analysis will be critical to address such technological challenges.

TABLE II. TABLE TyPe Styles

\begin{tabular}{|l|l|}
\hline Challenge & Description \\
\hline Heterogeneity & $\begin{array}{l}\text { The format of the data generated by different data } \\
\text { sources is a technological challenge that will require 1) } \\
\text { the homogenization of data formats and 2) the use of } \\
\text { gateways in order to address data transformation }\end{array}$ \\
\hline Integration & $\begin{array}{l}\text { The integration of isolated systems will force the } \\
\text { creation of new and adaptable interfaces between } \\
\text { systems. In addition, the integration of legacy systems } \\
\text { may be problematic for retrieving/receiving signals, data } \\
\text { and information to/from different interested parties. }\end{array}$ \\
\hline Interoperability & $\begin{array}{l}\text { Besides the issues at the interface, systems must } \\
\text { communicate within similar protocols. Mutual platforms } \\
\text { that are agnostic to protocols, such as OPC-UA may be } \\
\text { of use for the implementation of CPSoS. }\end{array}$ \\
\hline Security & $\begin{array}{l}\text { Whenever the integration of sensitive systems through } \\
\text { ICT, data security is a challenge. The proposed CPSoS } \\
\text { must ensure that the data which is exchanged by different } \\
\text { system cannot be accessed with malicious interests or, } \\
\text { simply, without permission }\end{array}$ \\
\hline
\end{tabular}

\section{CONCEPTUAL PROOF OF CONCEPT}

\section{A. Use case definition for implementing an industrial CPSoS}

One way of exploiting the potential of CPSoS for saving development cost and time, reducing complexity and making easier for innovators to realize new systems is the integration of systems. This research work proposes the integration of logistics, building automation and production systems in order to create a CPSoS with the ultimate goal of enhancing the quality and efficiency of industrial systems production. This section presents a possible scenario that will serve as a testbed for implementing the concept described in Section III. In this context there is a need to have a production system located at certain facilities with a deployed building conditioning system and the possibility to connect such systems with logistics.

The adoption of a CPSoS can be addressed using the following sequences of steps for connecting three main CPSoS subsystems (production, building and logistics systems). First, within the integration of two individual systems of the Factory Automation Systems and Technologies Laboratory (FASTLab.) located at Tampere University of Technology (TUT) facilities: 1) the FASTory area including a production line, a material preparation multi-robot cells and a storage zone, all forming a complete production system and 2) a set of devices deployed in both indoor and outdoor of the FAST-Lab, as a building automation system. Finally, it will also include the logistics system (LS) that permit the traceability of parts that are delivered to and needed by the FAST-Lab. production system.

The first CPSoS subsystem is a Building Automation System (BAS) composed by multiple hardware and software 
components. Figure 2 shows the entrance of the FASTory-Lab. facilities, marked as (1), has a set of LED lamps marked as (2) and (3) that light the area for a better material unloading within the detection of movement through an Onninen ${ }^{2}$ presence detector marked as (4), which is connected to an intelligent lighting controller from Valopaa $\mathrm{Oy}^{3}$. Due to its geographical location, Finland has a lack of light at certain months of the year. Thus, the use of outdoor efficient lighting facilitates the delivery of material for the production line, marked as (6). In addition, the BAS controls similar LED lamps deployed indoor and an Alfa Laval ${ }^{4}$ finned coil air heater, marked as (5), which will turn on when the inside temperature drops, due to the weather conditions in Finland.

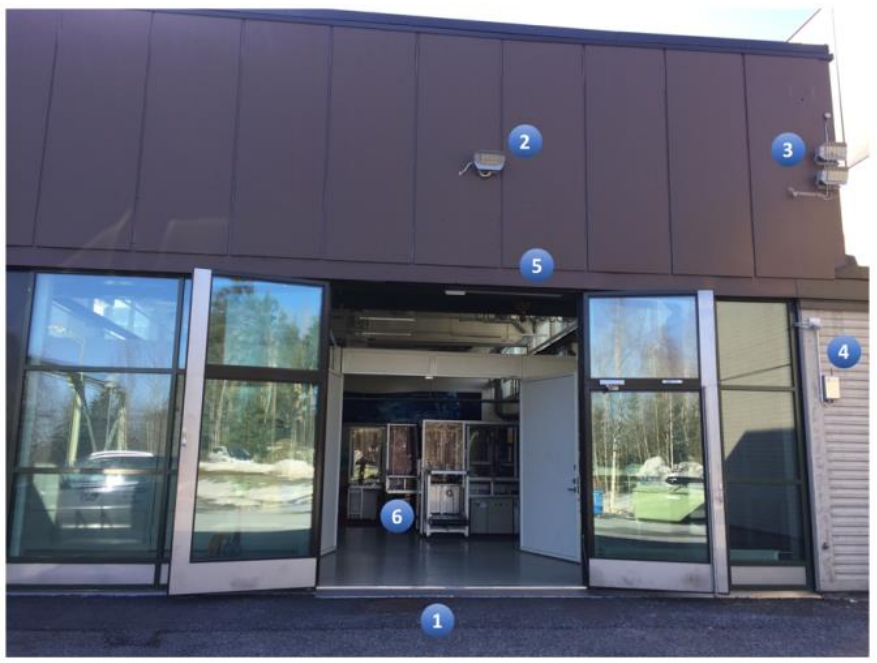

Figure 2. Outdoor of the FAST-Lab. TUT facilities

The BAS is monitored and controlled within web-service enabled controllers that are shown on the left side of Figure 3, marked as (A). More precisely, these controllers are the Inico S1000 Industrial controllers 5 , which are connected to a hub, marked as (B), for its configuration throughout the same network. Previous research demonstrates how to use such controllers considering energy-efficiency for LED-based lighting of multipurpose outdoor environments [55]. Moreover, the industrial controllers are connected through Wi-Fi to Temperature, Humidity and Light (THL) sensors which are strategically placed around all the facility for monitoring such type of measurements. This permits an energy efficient utilization of building temperature and light conditioning. The fact of using web services for monitoring and controlling physical equipment, defines this BAS as a CPS.

The second CPSoS subsystem is a Production System (PS) composed by different systems/areas: the FASTory production line, three multi-robotic cells for material preparation and the storage area for storing both parts and ready products for shipment. The PS is presented in Figure 4. Similar to the BAS, the FASTory production line, as well as the material preparation cells are controlled within the S1000 controllers and it is possible to remotely invoke service operations that may lead to the execution of physical tasks and/or monitoring specific information. The deployment of such devices is shown on the right side of Figure 3, marked as (A).
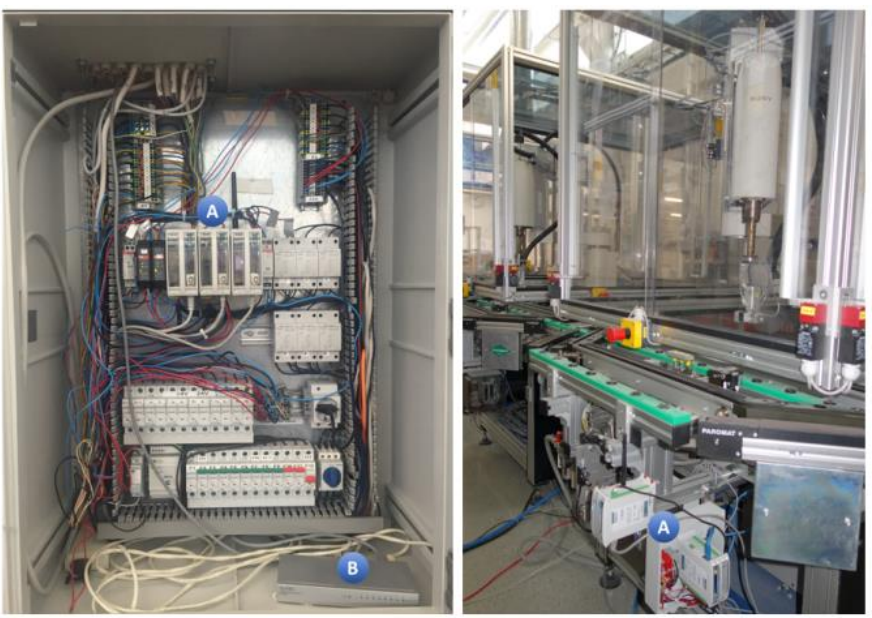

Figure 3. Industrial controllers, devices and connections for monitoring and controlling building automation and production systems at TUT facilities

A detailed description of the FASTory Production Line is shown in [56]. In terms of production process, the FASTory Production Line produces drawings of different mobile phones. Since the SCARA robots of the production line are programmed with different operations to draw several mobile parts that, in turn, can be painted within different colors, the line can produce more than seven hundred twenty mobile phones variants. The line is composed by 10 identic WorkStations (WS), i.e., WS2WS6 and WS8-WS12 for drawing and pallet transport operations, one WS for loading parts and unloading finished products, i.e., WS1 and one WS for manual palletization of the line. In addition, for this research work, pallets and papers are handled in the material preparation zone, within multi-robotic cells, i.e., WSA, WSB and WSC.

The last CPSoS subsystem is focused on monitoring, covering and synchronizing the logistic chain. In the proposed scenario, the productivity indices, storage capability, maintenance system and delivery time will be jointly considered to provide a complete traceability of the operations demanded to generate a product, event or service. Besides, the elements present in the subsystems interchange data/information to complete the sequence of tasks needs to cover the full production line, as well as, the dynamic monitoring and management of the delivery of produced part and the stock of component for predictive maintenance actions.

By the other hand, a real-time tracking system based on the combination of the mobile network information with localization technologies (Global Positioning Systems, GPS) will be synchronize BAS subsystem; collecting and providing information/data from and to the external delivery system to control the light, cooler and doors in function to the proximate of the pickup/delivery vehicle.

\footnotetext{
${ }^{2}$ https://onnshop.onninen.fi/

${ }^{3}$ https://lumous.fi/
}

\footnotetext{
${ }^{4}$ https://www.alfalaval.com/

${ }^{5} \mathrm{http}: / / \mathrm{www}$. inicotech.com/
} 


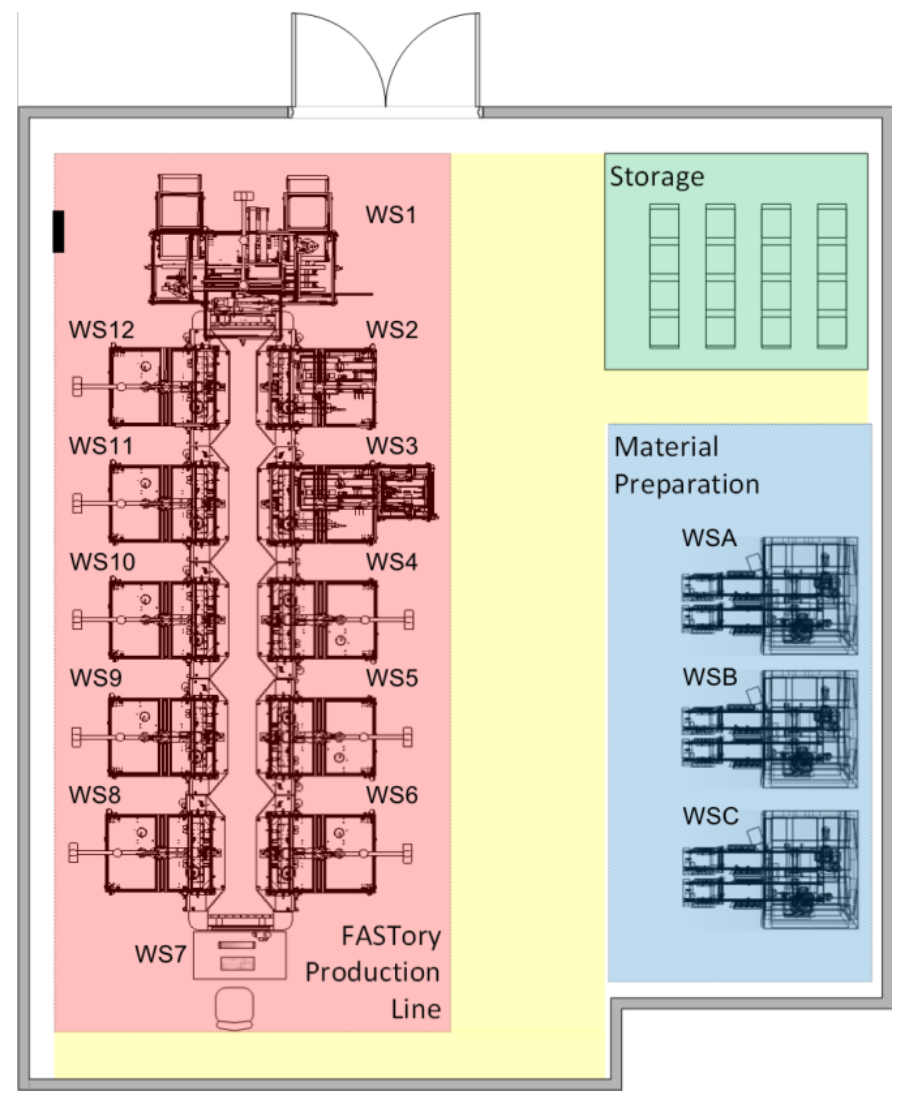

Figure 4. The FASTory area including a complete production scenario

\section{B. Expected results}

It is expected that the CPSoS will enhance the efficiency of the PS productivity throughout the integration of the information that is produced by currently isolated systems, i.e., BAS, PS and LS. Such integration will permit a rapid and automated reconfiguration of the PS due to required sudden changes that might affect the production plan. For example, production can be affected by changes on client demands, forecast changes or changes in the production resources. Currently, the three systems that will compose the CPSoS are loosely connected since each system does not consume any information from the other ones. This results with higher level of precautions that can be costlier, and less efficient. For instance, the storage shown in Figure 4 is always over filled with materials (pens and papers) that are not urgently needed because of the lack of awareness about the logistics and customer needs.

This envisioned approach can be validated using some of the Key Performance Indicators (KPIs) form ISO 22400-2 Standard [57]. In this manner, the presented approach might show less Storage and Transportation Loss Ratio (STLR) KPI since the storage will have less materials that can be expired (in the case of FASTory PS, pens are getting dry very fast on the shelf). Another ISO 22400-2 standard KPI is the Comprehensive Energy Consumption (CEC) KPI. This KPI is expected to decrease since the BA system can be aware about the shipment schedules, which in turn, it maintains the lighting and the heating at the optimum level, controlling the energy efficiency.

From a high-level perspective, the proposed scenario for implementing a CPSoS will integrate all sub-systems that are required to monitor and control production from delivering parts up to the shipment of the product, ensuring an efficient utilization of energy at production facilities.

\section{CONCLUSION}

This article presents some key steps toward the adoption of a Cyber-Physical Systems of Systems on industrial scenarios. The orchestration of three main systems (production, building automation and logistics) that are present in almost all industrial environments is considered. Furthermore, a group of new functionalities based on advanced data analytics, machine-tomachine IoT-based protocols, machine learning and knowledge representation algorithms in cloud-based platform are identified. This new digital functionality is essential to enable real-time information sharing and subsystems automation interaction from product design to logistics system, increasing the traceability levels for the entire value chain in industrial scenarios, as well as, the management and optimization of the quality of facility services, safety and energy efficiency and industrial productivity. In addition, this work presents one of way of validating the selected technologies, as well as, the verification of the presented CPSoS paradigm in a real industrial scenario by the FASTory production line, becoming a proper demonstrator/pilot for future Industry 4.0 use case.

\section{REFERENCES}

[1] A. Villalonga, G. Beruvides, F. Castaño, and R. Haber, "Industrial Cyber-Physical System for Condition-based Monitoring in Manufacturing Processes," in ICPS 2018 - 1st. International Conference on Industrial Cyber Physical Systems, 2018, pp. -.

[2] A. Villalonga, G. Beruvides, F. Castaño, and R. Haber, "ConditionBased Monitoring Architecture for CNC Machine Tools Based on Global Knowledge," in INCOM 2018 - 16th IFAC Symposium on Information Control Problems in Manufacturing, 2018, pp. -.

[3] bIoTope, "Building an IoT OPen innovation Ecosystem for connected smart objects," Horizon 2020 Research and Innovation Programme under grant agreement No. 688203, vol. 2016-2018.

[4] IoSENSE, "Flexible FE/BE Sensor Pilot Line for the Internet of Everything," Horizon 2020 Research and Innovation Programme. Electronic Component Systems for European Leadership Joint Undertaking under grant agreement No 692480, vol. 2016-2019.

[5] M. Jamshidi, System of Systems Engineering: Innovations for the 21st Century (System of Systems Engineering: Innovations for the $21<$ sup $>$ st $</$ sup $>$ Century). 2008, pp. $1-591$.

[6] C. Keating et al., "System of systems engineering," EMJ - Engineering Management Journal, Article vol. 15, no. 3, pp. 36-45, 2003.

[7] H. Wang, L. Wang, Q. Yu, Z. Zheng, and Z. Yang, "A proactive approach based on online reliability prediction for adaptation of serviceoriented systems," Journal of Parallel and Distributed Computing, Article vol. 114, pp. 70-84, 2018.

[8] G. Beruvides, C. Juanes, F. Castaño, and R. E. Haber, "A self-learning strategy for artificial cognitive control systems," in Proceeding - 2015 IEEE International Conference on Industrial Informatics, INDIN 2015, 2015, pp. 1180-1185.

[9] S. Iarovyi, J. L. M. Lastra, R. Haber, and R. Del Toro, "From artificial cognitive systems and open architectures to cognitive manufacturing systems," in Proceeding - 2015 IEEE International Conference on Industrial Informatics, INDIN 2015, 2015, pp. 1225-1232. 
[10] I. La Fe, G. Beruvides, R. Quiza, R. Haber, and M. Rivas, "Automatic selection of optimal parameters based on simple soft computing methods. A case study on micro-milling processes," IEEE Transactions on Industrial Informatics, Article in Press 2018.

[11] H. Kagermann, J. Helbig, A. Hellinger, and W. Wahlster, Recommendations for implementing the strategic initiative INDUSTRIE 4.0: Securing the future of German manufacturing industry; final report of the Industrie 4.0 Working Group. Forschungsunion, 2013.

[12] M. Wollschlaeger, T. Sauter, and J. Jasperneite, "The future of industrial communication: Automation networks in the era of the internet of things and industry 4.0," IEEE Industrial Electronics Magazine, vol. 11, no. 1, pp. 17-27, 2017.

[13] B. Sniderman, M. Mahto, and M. J. Cotteleer, "Industry 4.0 and manufacturing ecosystems: Exploring the world of connected enterprises," Deloitte Consulting, 2016.

[14] M. Elshenawy, B. Abdulhai, and M. El-Darieby, "Towards a serviceoriented cyber-physical systems of systems for smart city mobility applications," Future Generation Computer Systems, Article vol. 79, pp. $575-587,2018$

[15] X. Hu, T. H. S. Chu, H. C. B. Chan, and V. C. M. Leung, "Vita: A crowdsensing-oriented mobile cyber-physical system," IEEE Transactions on Emerging Topics in Computing, Article vol. 1, no. 1, pp. 148-165, 2013, Art. no. 6558754

[16] A. A. F. Saldivar, Y. Li, W. N. Chen, Z. H. Zhan, J. Zhang, and L. Y. Chen, "Industry 4.0 with cyber-physical integration: A design and manufacture perspective," in 2015 21st International Conference on Automation and Computing: Automation, Computing and Manufacturing for New Economic Growth, ICAC 2015, 2015.

[17] M. Ramírez, R. Haber, V. Peña, and I. Rodríguez, "Fuzzy control of a multiple hearth furnace," Computers in Industry, Article vol. 54, no. 1 , pp. 105-113, 2004

[18] R. E. Precup, P. Angelov, B. S. J. Costa, and M. Sayed-Mouchaweh, "An overview on fault diagnosis and nature-inspired optimal control of industrial process applications," Computers in Industry, Article vol. 74, pp. 1-16, 2015.

[19] G. Beruvides, F. Castaño, R. E. Haber, R. Quiza, and A. Villalonga, "Coping with Complexity When Predicting Surface Roughness in Milling Processes: Hybrid Incremental Model with Optimal Parametrization," Complexity, Article vol. 2017, 2017, Art. no. 7317254.

[20] C. Thuemmler and C. Bai, Health 4.0: How virtualization and big data are revolutionizing healthcare. Springer, 2017.

[21] T. Nanayakkara, F. Sahin, and M. Jamshidi, Intelligent Control Systems with An Introduction to System of Systems Engineering. CRC Press, 2010 .

[22] C. A. Lana, N. M. Souza, M. E. Delamaro, E. Y. Nakagawa, F. Oquendo, and J. C. Maldonado, "Systems-of-systems development: Initiatives, trends, and challenges," in 2016 XLII Latin American Computing Conference (CLEI), 2016, pp. 1-12.

[23] I. G. Vargas, T. Gottardi, and R. T. V. Braga, "Approaches for Integration in System of Systems: A Systematic Review," in 2016 IEEE/ACM 4th International Workshop on Software Engineering for Systems-of-Systems (SESoS), 2016, pp. 32-38.

[24] J. Godoy, J. Pérez, E. Onieva, J. Villagrá, V. Milanés, and R. Haber, "A driverless vehicle demonstration on motorways and in urban environments," Transport, Article vol. 30, no. 3, pp. 253-263, 2015.

[25] A. Artuñedo, R. M. Del Toro, and R. E. Haber, "Consensus-based cooperative control based on pollution sensing and traffic information for urban traffic networks," Sensors (Switzerland), Article vol. 17, no. 5, 2017, Art. no. 953

[26] M. Antal, C. Pop, T. Cioara, I. Anghel, I. Salomie, and F. Pop, "A System of Systems approach for data centers optimization and integration into smart energy grids," Future Generation Computer Systems, 2017/05/24/ 2017.

[27] W. L. Barnes Ii et al., "Coordination of Constituent Systems for Functionalizing Systems of Systems: An Exploration," Procedia Computer Science, vol. 114, pp. 375-383, 2017/01/01/2017.

[28] A. C. Tundrea, C. Draganescu, and C. Popa, "Integrating Intelligent Transport Systems in a Risk Management System of Systems," in 2017 21st International Conference on Control Systems and Computer Science (CSCS), 2017, pp. 385-391.
[29] R. E. Haber, G. Beruvides, R. Quiza, and A. Hernandez, "A simple multiobjective optimization based on the cross-entropy method," IEEE Access, Article vol. 5, pp. 22272-22281, 2017, Art. no. 8070310.

[30] G. Beruvides, R. Quiza, and R. E. Haber, "Multi-objective optimization based on an improved cross-entropy method. A case study of a microscale manufacturing process," Information Sciences, Article vol. 334335, pp. 161-173, 2016

[31] K. Kawakami, S. Fukushige, and H. Kobayashi, "A Functional Approach to Life Cycle Simulation for System of Systems," Procedia CIRP, vol. 61, pp. 110-115, 2017/01/01/2017.

[32] L. M. Kritzinger, T. Krismayer, M. Vierhauser, R. Rabiser, and P. Grünbacher, "Visualization support for requirements monitoring in systems of systems," in 2017 32nd IEEE/ACM International Conference on Automated Software Engineering (ASE), 2017, pp. 889-894.

[33] R. E. Haber, C. Juanes, R. del Toro, and G. Beruvides, "Artificial cognitive control with self-x capabilities: A case study of a micromanufacturing process," Computers in Industry, vol. 74, pp. 135-150, 2015/12/01/ 2015 .

[34] S. Lucia, M. Kögel, P. Zometa, D. E. Quevedo, and R. Findeisen, "Predictive control, embedded cyberphysical systems and systems of systems - A perspective," Annual Reviews in Control, vol. 41, pp. 193 207, 2016/01/01/2016.

[35] E. B. Kosmatopoulos, I. Michailidis, C. D. Korkas, and C. Ravanis, "Local4Global Adaptive Optimization and control for System-ofSystems," in 2015 European Control Conference (ECC), 2015, pp. 35363541.

[36] A. W. Colombo, T. Bangemann, and S. Karnouskos, "A system of systems view on collaborative industrial automation," in 2013 IEEE International Conference on Industrial Technology (ICIT), 2013, pp. 1968-1975.

[37] A. Morkevicius, L. Bisikirskiene, and G. Bleakley, "Using a systems of systems modeling approach for developing Industrial Internet of Things applications," in 2017 12th System of Systems Engineering Conference (SoSE), 2017, pp. 1-6.

[38] T. Samad and T. Parisini, "Systems of Systems," in The Impact of Control Technology, T. Samad and A. M. Annaswamy, Eds. available at www.ieeecss.org: IEEE Control Systems Society, 2011, pp. 175-183.

[39] M. Rüßmann et al., "Industry 4.0: The future of productivity and growth in manufacturing industries," Boston Consulting Group, vol. 9, 2015.

[40] P. Zheng et al., "Smart manufacturing systems for Industry 4.0 Conceptual framework, scenarios, and future perspectives," Frontiers of Mechanical Engineering, Review vol. 13, no. 2, pp. 137-150, 2018.

[41] A. W. Colombo et al., Industrial cloud-based cyber-physical systems: The IMC-AESOP approach (Industrial Cloud-Based Cyber-Physical Systems: The IMC-AESOP Approach). 2014, pp. 1-245.

[42] B. Ramis Ferrer and J. L. Martinez Lastra, "Private local automation clouds built by CPS: Potential and challenges for distributed reasoning," Advanced Engineering Informatics, vol. 32, pp. 113-125, 2017/04/01/ 2017.

43] B. R. Ferrer and J. L. M. Lastra, "An architecture for implementing private local automation clouds built by CPS," in IECON 2017 - 43rd Annual Conference of the IEEE Industrial Electronics Society, 2017, pp. 5406-5413.

[44] N. K. Verma, T. Sharma, S. Maurya, D. J. Singh, and A. Salour, "Realtime monitoring of machines using Open Platform Communication," in 2017 IEEE International Conference on Prognostics and Health Management, ICPHM 2017, 2017, pp. 124-129.

[45] H. Flatt, S. Schriegel, J. Jasperneite, H. Trsek, and H. Adamczyk, "Analysis of the Cyber-Security of industry 4.0 technologies based on RAMI 4.0 and identification of requirements," in IEEE International Conference on Emerging Technologies and Factory Automation, ETFA, 2016, vol. 2016-November.

[46] H. Fleischmann, M. Brossog, M. Beck, and J. Franke, "Automated generation of human-machine interfaces in electric drives manufacturing," in 2017 7th International Electric Drives Production Conference (EDPC), 2017, pp. 1-8.

[47] S. Iarovyi, W. M. Mohammed, A. Lobov, B. R. Ferrer, and J. L. M. Lastra, "Cyber\&\#x2013;Physical Systems for Open-Knowledge-Driven Manufacturing Execution Systems," Proceedings of the IEEE, vol. 104, no. 5, pp. 1142-1154, 2016 
[48] B. Ramis Ferrer and J. L. Martinez Lastra, "Towards the encapsulation and decentralisation of OKD-MES services within embedded devices," International Journal of Production Research, pp. 1-13, 2017.

[49] E. Arica and D. Powell, "Status and future of manufacturing execution systems," in Industrial Engineering and Engineering Management (IEEM), 2017 IEEE International Conference on, 2017, pp. 2000-2004: IEEE.

[50] M. El Mohadab, B. B. Khalene, and S. Safi, "Enterprise resource planning: Introductory overview," in Electrical and Information Technologies (ICEIT), 2017 International Conference on, 2017, pp. 1-4: IEEE.

[51] L. Ranieri, S. Digiesi, B. Silvestri, and M. Roccotelli, "A Review of Last Mile Logistics Innovations in an Externalities Cost Reduction Vision," Sustainability, vol. 10, no. 3, p. 782, 2018.

[52] T. Vafeiadis, D. Ioannidis, C. Ziazios, I. N. Metaxa, and D. Tzovaras, "Towards Robust Early Stage Data Knowledge-based Inference Engine to Support Zero-defect Strategies in Manufacturing Environment," Procedia Manufacturing, vol. 11, pp. 679-685, 2017/01/01/ 2017.
[53] Z. Sheng, C. Mahapatra, C. Zhu, and V. C. M. Leung, "Recent Advances in Industrial Wireless Sensor Networks Toward Efficient Management in IoT," IEEE Access, vol. 3, pp. 622-637, 2015.

[54] B. R. Ferrer, S. O. Afolaranmi, and J. L. M. Lastra, "Principles and risk assessment of managing distributed ontologies hosted by embedded devices for controlling industrial systems," in IECON 2017 - 43rd Annual Conference of the IEEE Industrial Electronics Society, 2017, pp. 3498-3505.

[55] A. Farahat, A. Florea, J. L. M. Lastra, C. Brañas, and F. J. A. Sánchez, "Energy Efficiency Considerations for LED-Based Lighting of Multipurpose Outdoor Environments," IEEE Journal of Emerging and Selected Topics in Power Electronics, vol. 3, no. 3, pp. 599-608, 2015.

[56] W. M. Mohammed, A. Lobov, B. R. Ferrer, S. Iarovyi, and J. L. M. Lastra, "A web-based simulator for a discrete manufacturing system," in IECON 2016 - 42nd Annual Conference of the IEEE Industrial Electronics Society, 2016, pp. 6583-6589.

[57] ISO 22400-2:2014 Automation systems and integration -- Key performance indicators (KPIs) for manufacturing operations management -- Part 2: Definitions and descriptions, 2014 